\title{
Potencialidades y desafíos del Turismo y ecoturismo en el esta- do de San Luis Potosí, México: retos y expectativas para alcanzar el desarrollo regional ${ }^{1}$
}

\author{
José Guadalupe Rivera González ${ }^{\text {i }}$ \\ Valente Vázquez Solís ii \\ Humberto Reyes Hernández ${ }^{\text {iii }}$ \\ Óscar Reyes Pérez iv \\ Miguel Nicolas Caretta ${ }^{v}$
}

Universidad Autónoma de San Luis Potosí (México)

\begin{abstract}
Resumen: El objetivo del trabajo es analizar la presencia de diversos actores y componentes en el estado de San Luis Potosí, México que pueden potenciar y que a la vez también que pueden ser vistos como obstáculos para que el turismo y ecoturismo logren convertirse en actividades que promuevan el bienestar económico y el desarrollo de cada una de las 4 regiones que conforman el estado. La investigación se sustenta en trabajo de campo, entrevistas con funcionarios municipales relacionados con el turismo, delegados regionales de la secretaria de turismo en las regiones Media, Altiplano y Huasteca del estado y con prestadores de servicios.
\end{abstract}

Palabras clave: Turismo; Ecoturismo; San Luis Potosí; Desarrollo regional.

Title: Potentialities and challenges of the Tourism and ecoturismo in the state of San Luis Potosí, Mexico: challenges and expectations to reach the regional development.

\begin{abstract}
The objective of the work is analyze the presence of diverse actors and components in the state of San Luis Potosí, Mexico that can harness and that simultaneously also that can be seen like obstacles so that the tourism and ecoturismo manage to become activities that promote the economic well-being and the development of each one of the regions of the State. The investigation is sustained in work of field, interviews with municipal civil employees related to the tourism, regional delegates of the secretary of tourism in the regions Media, Altiplano and Huasteca of the serve with lenders.
\end{abstract}

Keywords: Cultural Tourism, Identity; Performance; Bumba-meu-boi; São Luís do Maranhão.

\footnotetext{
i Profesor-Investigador de tiempo completo de la Coordinación de Ciencias Sociales y Humanidades de la Universidad Autónoma de San Luis Potosí/ Dr. en Antropología. joserivera@uaslp.mx

ii Profesor-Investigador de tiempo completo de la Coordinación de Ciencias Sociales y Humanidades de la Universidad Autónoma de San Luis Potosí/ Dr. en Geografía. vasquezsv@uaslp.mx

iii Profesor-Investigador de tiempo completo de la Coordinación de Ciencias Sociales y Humanidades de la Universidad Autónoma de San Luis Potosí/ Dr. en Geografía. hreyes@uaslp.mx

iv Profesor-Investigador de tiempo completo de la Coordinación de Ciencias Sociales y Humanidades de la Universidad Autónoma de San Luis Potosí/ Dr. en Geografía. osrp@uaslp.mx

v Profesor-Investigador de tiempo completo de la Coordinación de Ciencias Sociales y Humanidades de la Universidad Autónoma de San Luis Potosí/ Dr. en Arqueología.ncaretta@uaslp.mx
} 


\section{Introducción}

La sola existencia del patrimonio natural y cultural se ha visualizado, en muchas ocasiones, como elementos que por si mismos estarían en condiciones de contribuir a dinamizar el desarrollo humano en regiones económicamente deprimidas y vulnerables. Sin embargo, lograr que el patrimonio natural y cultural se transforme en elementos generadores de oportunidades de desarrollo económico y humano requiere también de la conjunción y voluntad de diversos actores y diversas instituciones, quienes a su vez representan y defienden diversos y complejos intereses. En este sentido, a partir de un intenso trabajo de investigación el cual contempló la realización de trabajo de campo realizado en 11 municipios del estado de San Luis Potosí, México, se estuvo en condiciones de conocer de primera mano el punto de vista de los actores y funcionarios locales, lo cual permitió tener conocimiento de los problemas y las contrariedades que cotidianamente enfrentan y que en algunas ocasiones imposibilitan el buen desarrollo y operación de programas oficiales o de proyectos impulsados desde y por las propias comunidades para el desarrollo del turismo y del ecotururismo. Finalmente, se concluye con una serie de contribuciones, desde la que los autores pretender contribuir al desarrollo de mejores acciones y programas que contribuyan a que se planifique de una mejor manera el desarrollo de las regiones y de las localidades considerando para ello no sólo el punto de vista de los expertos y de los funcionarios de primer nivel, sino también las experiencias y las inquietudes de los actores municipales y locales.

\section{El turismo bajo el análisis de las ciencias sociales}

Es un hecho que las ciencias sociales y en particular la antropología, la geografía y la sociología han contribuido en las últimas décadas, desde diferentes enfoques, al análisis del fenómeno del turismo en el contexto de las sociedades modernas y su vinculación-relación con otras actividades, además de su impacto y alcance global. En este sentido, algunos investigadores en tiempos recientes han destacado que la actividad turística es vista como una actividad global y que representa y expresa fielmente los procesos emergentes del modelo capitalista y la modernidad, es decir una serie de cambios estructurales y organizacionales en el sistema económico mundial, avance tecnológico en las comunicaciones, nuevas pautas de producción y consumo, así como formas de vida y concepciones culturales inéditas. Las líneas de investigación que se destacaron por los estudios desarrollados desde las ciencias sociales, se han centrado en el estudio de los turistas, las diversas relaciones que se establecían entre los turistas y las poblaciones nativas, los procesos de operacióndesarrollo del turismo y finalmente la serie de impactos de tipo económico y de tipo sociocultural entre las poblaciones o comunidades anfitrionas. Es importante destacar que en estas mismas investigaciones se privilegió también la mirada del turismo como una importante actividad ge- neradora de oportunidades y como una vía para alcanzar el desarrollo y el crecimiento económico.

No obstante los avances que se habían alcanzado en esta etapa de los estudios, era evidente la necesidad de analizar al fenómeno del turismo desde una perspectiva teórico-epistemológica que privilegiara una mirada integral del fenómeno. En este sentido, con el paso del tiempo empezaría a ganar terreno la perspectiva que haría de los actores locales actores más participativos y dejarían de ser vistos como meros actores pasivos que poco o nada se involucran y deciden en el proceso de toma de decisiones. A partir de nuevos enfoques, los investigadores empezaron a percibir al turismo como un fenómeno mucho más complejo y mucho más fragmentario, ya que no se trata únicamente de un proceso que involucrara los recursos naturales, culturales, sino que también involucra ámbitos económicos, políticos, culturales y territoriales. Es decir, el turismo se empezó a concebir como una actividad transformadora de los espacios, territorios y de los significados. Es decir se percibe como una actividad que involucra a un amplio número de instancias y actores sociales: viajeros, prestadores de servicios, gobiernos federales, estatales y municipales, residentes y además involucra, crea y transforma diversas formas y prácticas del consumo y de la representación cultural, generando importantes influencias en diversos ordenes entre todos los actores y procesos que están involucrados.

En los contextos de la llamada globalización, han sido diversos los acercamientos que se han realizado para analizar la experiencia del turismo y la construcción del proceso de mercantilización a su alrededor. Es decir, cómo ciertos bienes transforman su valor de uso por valor de cambio a partir de su inserción al mercado de la actividad turística. En este sentido, las investigaciones destacaron el proceso de producción de experiencias y de imágenes con fines recreativos y de diversión. Otros más vinieron a explicar la noción del turismo como una expresión de la modernidad del mundo capitalista y que ofrece sentido y significado a las actividades desarrolladas por los turistas. Otras investigaciones estuvieron explorando el proceso a través del cual algunas festividades religiosas en comunidades campesinas-indígenas se transforman en un importante atractivo para las agencias de turismo, quienes en algunos casos intervienen para montar una serie de actividades para atraer la atención de visitantes extranjeros, contribuyendo de esta manera a trastocar el sentido y el significado de dichas celebraciones. Así, el turismo se transformó entonces en una actividad planificada y enfocada para que el visitante pueda desarrollar una serie de experiencias que lo coloquen en la cercanía del mar, del bosque, del patrimonio histórico y de un conjunto de festividades culturales que le brindan al visitante una perspectiva de lejanía y de confort.

La amplia gama de investigaciones realizadas desde muy diferentes enfoques teóricos, en particular desde la antropología, la geografía y la sociología, nos permite entender que el turismo y sus amplias y complejas manifestaciones o ramificaciones han hecho sentir su influencia 
en los más variados y recónditos espacios y territorios alrededor del planeta. En ello se han involucrado a diferentes sectores y se han ampliado los recursos que se han hecho atractivos para el desarrollo de la actividad turística. Es decir, ya no sólo son los recursos naturales (Principalmente los destinos de playa) los que busca el visitante como la primera y única opción de descanso y de diversión. Cada vez se han hecho más presente el recurso cultural tangible y el intangible como una alternativa o como una opción para el desarrollo del turismo en comunidades étnicas y campesinas. De ahí se han desprendido diversas opciones como el llamado ecoturismo, el etnoturismo, el turismo comunitario, el agroturismo y otras vertientes más. De esta manera, las comunidades y las agencias privadas y las agencias gubernamentales han buscado alcanzar o promover distintas alternativas para alcanzar el crecimiento económico y lograr, a partir de la actividad turística, promover el bienestar de los países, las regiones y las comunidades locales (Marin Guardado, 2006; López Santillán y Marín Guardado, 2010).

\section{Algunos aspectos relevantes sobre la práctica del turismo y el ecoturismo}

Desde finales de los años ochenta del siglo XX, modalidades alternativas de turismo germinaron en naciones de Europa Occidental y en Norteamérica, en donde los modelos tradicionales de recreación masiva en sitios de playa y en zonas arqueológicas se consideraban obsoletos, inoperantes y sin propuestas novedosas para visitantes que buscaban satisfacer expectativas más selectivas, asociadas con la esencia del turismo antaño. El descanso, el retorno a la naturaleza y la concurrencia a sitios poco poblados se reconocieron como las características más valoradas por las novedosas formas de viaje que emergieron desde entonces (Vázquez Solís, 2008; Vázquez Solís y Benito del Pozo, 2009; Genet Guzmán Mauricio, 2008).

En el marco de un crecimiento económico sin precedentes del sector, el ecoturismo es la modalidad recreativa que ha experimentado el mayor desarrollo en el último decenio, tanto por el número de visitantes que se interesan en realizarlo, como por la diversificación de destinos, circuitos y espacios que se han creado y consolidado para este fin, además de que muestra una creciente complejidad orientada a satisfacer los gustos y necesidades de visitantes que reúnen un perfil mucho más variado. En el cuadro 1 se destacan los perfiles más relevantes de quienes practican el ecoturismo, bien sea por el interés, o por el comportamiento o relación que establecen con el sitio visitado.

De manera paralela a la clasificación expuesta, mucho se ha discutido sobre si concurrir a un sitio natural conlleva necesariamente una práctica responsable. El turismo "verde", que en sus inicios se planteó como la mejor alternativa para desarrollar una conciencia de conservación del ambiente, no es una actividad limitativa, pues tiene la capacidad de combinarse con otras modalidades tradicionales que exigen altos consumos de recursos naturales y derivan en impactos ambientales significativos, a través de la ejecución de actividades altamente depredadoras de la naturaleza, en tanto así, el ecoturismo se convierte en una actividad adicional, que lejos de sustituir prácticas añejas, promueve el consumo de espacios que permanecían ajenos a la concurrencia de turistas. Por lo tanto, uno de los retos más importantes del turismo de bajo impacto es consolidar una práctica verdaderamente responsable para quien lo practica, pero que al mismo tiempo integre un conjunto de productos y servicios atractivos, no solo para el visitante, sino que tenga la capacidad de generar ingresos económicos para la población local que reside en sitios ecoturísticos.

\section{La práctica del turismo y ecoturismo en México}

En México, el turismo representa una de las principales actividades económicas por la derrama de ingresos que representa para el país. Por ejemplo, en el año 2007, por este concepto, la economía del país tuvo ingresos por 12,900 millones de dólares, lo anterior representó un incremento del $6 \%$ en comparación con el 2006. Sobre el origen de los turistas internacionales que llegaron, el 67\% proviene de los Estados Unidos. Los ingresos del turismo se encuentran divididos en los centros de sol y playa, el turismo de aventura, los pueblos mágicos, el turismo social, de negocios y otras modalidades que se practican en otras áreas que buscan consolidarse como un destino atractivo para el turismo nacional como el internacional. Además el poco dinamismo del sector exportador y la industria petrolera nacional, así como la caída de las remesas provenientes de los migrantes en los Estados Unidos a partir de la crisis económica que se desató en septiembre de 2008, son elementos que han actuado para que los mayores ingresos económicos del país en los últimos años provengan de la actividad turística.

En el 2006, México ocupó el $8^{\circ}$ lugar en la captación de turistas extranjeros, registrando, en ese año, la llegada de 21.4 millones de turistas internacionales, y quedó ubicado entre los diez destinos predilectos por los turistas internacionales, quienes gastan en promedio 330-350 dólares por estancia. En este sentido, el turismo aparece como una importante actividad generadora no solamente de ingresos sino que también se ha conformado en una importante fuente de empleos en nuestro país. Tan solo en el año 2002, el empleo en este sector representó alrededor del $4 \%$ del total de la población económicamente activa. Se estima que la ocupación hotelera en México en los últimos años en promedio se encuentra entre el 60 y $80 \%$, dependiendo de la temporada vacacional, un número de cuartos disponibles cercano a los 500,000 (www.explorandomexico.com.mx/about-mexico/5/137).

México, contiene en sí mismo una gran diversidad de recursos naturales y culturales que lo hace naturalmente un destino turístico potencialmente importante para el desarrollo de la economía. Posee más de 3,000 Kilómetros de frontera común con los Estados Unidos. Además es un centro económico, social, político y cultural apto para este 
tipo de actividad. En este sentido, destacan las siguientes modalidades del turismo como algunas de las más significativas que se presentan y desarrollan de manera cotidiana:

- El turismo social. Esta modalidad tiene un fuerte rezago, toda vez que los ingresos de las instituciones nacionales que promovían este tipo de ofertas a sus trabajadores, se encuentran en severas crisis económicas, entiéndase Instituto Mexicano del Seguro Social (IMSS) y el Instituto de Seguridad y Servicios Sociales de los Trabajadores del Estado (ISSSTE). Al mismo tiempo el país cuenta con alternativas de ciudades coloniales como: Taxco, Guanajuato, Zacatecas y la propia ciudad de San Luis Potosí.

- Turismo de la tercera edad y jubilados. Junto con el área de turismo social, es un rubro sumamente deficiente. Es importante destacar que en el Plan Nacional de Desarrollo 2007-2012, los programas sociales de atención a la tercera edad se encuentran con una reducción importante del presupuesto anual autorizado en la Cámara de Diputados. Esto significa que los programas turísticos para jubilados sólo están siendo apoyados por albergues. No ha podido llegarse a un acuerdo en donde haya un descuento significativo y generalizado por parte de los prestadores de servicios hoteleros.

- Turismo naturista. Este es uno de los rubros que se proyecta con mayor crecimiento en los próximos años. En este sentido, para el 2008 se tuvo proyectado un crecimiento del $12 \%$ en las zonas turísticas de alta demanda como Acapulco, Cancún, Vallarte y los Cabos. Además de que en los últimos años han mostrado un importante crecimiento otros sitios, como las aguas sulfurosas de Atonaltzin y Zinapécuaro, ambos localizados en el estado de Michoacán, formando parte de la ruta turística de la salud que se ofrece en el estado, otro ejemplo es la región Huasteca en el estado de San Luis Potosí.

- Turismo de playa. Este es uno de los sectores de mayor dinamismo, ya que sitios como: Cancún, Playa del Carmen, Cozumel ,Chetumal, Nuevo Vallarta-Vallarta, Manzanillo, Puerto Escondido, Huatulco, Acapulco-Ixtapa Mazatlan, Guaymas, La Paz, San José del Cabo y Cabo San Lucas, siguen siendo los destinos en donde se recibe el $82 \%$ de los turistas que provienen del extranjero (www.explorandomexico.com.mx/about México/5/137/).

Aunque en algunos países de Norteamérica, Europa y Asia Sur y Nororiental existe una tradición añeja por este tipo de turismo, en México se ha implementado de forma incipiente, y se reduce a lugares en donde se han promocionado circuitos que ofertan descenso por algunos ríos, observación de flora y fauna, rappel, senderismo, montañismo; actividades realizadas preferentemente por los visitantes procedentes de aquellas regiones del mundo. Sin embargo, y aunque el turismo alternativo o de bajo impacto puede practicarse en zonas naturales protegidas, la inseguridad para el turista derivado de la incursión a este tipo de lugares constituye, por ahora, una limitante para que esta práctica se difunda a un mayor número de sitios en México.

Debido a la necesidad de implementar nuevas modalidades de turismo que respondan, no sólo a las expectativas de los visitantes que buscan el contacto con ambientes naturales poco modificados por la acción humana, sino de promover la incorporación de la población residente en los sitios con aprovechamiento turístico potencial, es preciso observar las ventajas asociadas con una de las modalidades de práctica turística que han adquirido mayor relevancia en los últimos años: el ecoturismo (Sánchez, 2001).

No obstante el crecimiento turístico que el país ha evidenciado en el último cuarto de siglo, la actividad no ha resultado la panacea como muchos parecen pensar. En este mismo sentido puede destacar el caso particular de los puertos de Acapulco y Cancún. En ambos lugares se reflejan los radicales contrastes entre un mundo de lujo y comodidad y el de grandes necesidades y carencias. La infraestructura de primer mundo de algunos de estos destinos turísticos contrasta de manera significativa con las condiciones de atraso, pobreza y marginación de algunas zonas aledañas a estos prestigiados y caros lugares. Son estas colonias populares en donde habita gran parte de la mano de obra de hoteles, restaurantes, tiendas de autoservicios quienes conviven diariamente con una realidad diferente, donde predomina la pobreza y escasean los servicios básicos. Los anteriores son apenas dos ejemplos, entre muchos más que ilustran la degradación ambiental, inseguridad y la agudización de problemas sociales como drogadicción, prostitución y, por supuesto, el narcotráfico (Rivera González, 2005).

Es indudable que destinos turísticos como Acapulco y Cancún y muchos otros centros turísticos en el país contribuyen a generar una importante cantidad de recursos económicos, sin embargo la paradoja es que esos millonarios recursos no se utilizan para solucionar las carencias o para mejorar las condiciones de vida de los habitantes y trabajadores locales. Sobre esto último, la Secretaría de Desarrollo Social (SEDESOL), determinó que las ciudades turísticas y petroleras del país -donde se genera 10\% de la riqueza nacional- son a la vez poseedoras de altos índices de pobreza urbana y marginación social, entre ellos proliferación de asentamientos irregulares, debilitamiento del tejido social y falta de atención a problemas sociales por parte de las autoridades. Las cifras respecto a las divisas que genera el turismo son contundentes, sin embargo, no bastan para legitimar y asumir automáticamente que esta economía es el motor del desarrollo (Marín Guardado, 2006).

\section{Potencial del turismo y ecoturismo en el estado de San Luis Potosí, México}

El estado de San Luis Potosí, se localiza entre las coordenadas geográficas extremas $21^{\circ} 10^{\prime}$ y $24^{\circ} 30^{\prime}$ latitud norte, y $98^{\circ} 20^{\prime}$ y $102^{\circ} 20^{\prime}$ longitud oeste en la porción central de México. El estado potosino ocupa una parte de la 


\begin{tabular}{|c|c|c|}
\hline Perfil de visitante & Características y prácticas frecuentes & Sitios concurridos \\
\hline Aventurero & $\begin{array}{l}\text { Involucra una participación activa del visitante en } \\
\text { la medida que demanda un conjunto de condiciones } \\
\text { físicas para su práctica. Forma parte del reto de } \\
\text { quienes practican el montañismo, la escalada libre, } \\
\text { el ciclismo de montañay el kayak. }\end{array}$ & $\begin{array}{l}\text { Cordilleras del } \\
\text { Himalaya, Alpes y } \\
\text { Andes, Cañón del } \\
\text { Colorado. }\end{array}$ \\
\hline Contemplativo & $\begin{array}{l}\text { En esta categoria se inscribe un amplio perfil de } \\
\text { visitantes por la motivación que los anima a } \\
\text { concumir a sitios naturales, pero en general, se } \\
\text { distingue por una actitud relativamente pasiva } \\
\text { ligada al descanso y la actividad moderada. } \\
\text { Ejerrylo de lo anterior es el turismo "familiar" que } \\
\text { efectúa caminata, paseos y juegos recreativos en } \\
\text { áreas abiertas, la pintura de los paisajes }\end{array}$ & $\begin{array}{l}\text { Parques naturales, } \\
\text { reservas de la biosfera, } \\
\text { paisajes espectaculares } \\
\text { en barrancas o } \\
\text { montañas. }\end{array}$ \\
\hline Conocedor de la naturaleza & $\begin{array}{l}\text { Representa al perfil de visitantes que, por el amplio } \\
\text { conocimiento que poseen de los espacios naturales, } \\
\text { corresponde con quienes por lo general realizan } \\
\text { prácticas responsables. Se incluyen a los } \\
\text { científicos, admiradores de la naturaleza o personas } \\
\text { que han desarrollado una conciencia empínica sobre } \\
\text { el cuida do del ambiente. }\end{array}$ & $\begin{array}{l}\text { Incluye cualquier } \\
\text { espacio natural } \\
\text { correspondiente a las } \\
\text { demás modalidades de } \\
\text { visitantes. }\end{array}$ \\
\hline Ocasional & $\begin{array}{l}\text { En esta categoria se reconocen a los visitantes que } \\
\text { de forma circunstancial concurren a lugares } \\
\text { ecoturísticos, bien porque son "iconos curya visita } \\
\text { no se puede obviar", o porque fomman parte de } \\
\text { destinos secundanios para los intereses de los } \\
\text { turistas, pero que se inchyen en circuitos } \\
\text { organizados por turoperadoras o agencias de viajes. } \\
\text { A razón de lo anterior, al no ser destinos } \\
\text { predilectos para los visitantes de esta categonia y } \\
\text { que prefieren otras altemativas, el tiempo de } \\
\text { permanencia en estos lugares es bastante corto. }\end{array}$ & $\begin{array}{l}\text { Además de los } \\
\text { descritos en el turismo } \\
\text { aventurero, se } \\
\text { incluyen el Glaciar } \\
\text { Perito Moreno, las } \\
\text { Cataratas de Iguazí y } \\
\text { las delNiágara. }\end{array}$ \\
\hline
\end{tabular}

Cuadro 1. Clasificación de prácticas ecoturísticas recurren.

Altiplanicie Mexicana cuenta con una superficie total de $61,161 \mathrm{~km} 2$ y está dividido en cuatro grandes regiones que son Altiplano, Centro, Media y Huasteca. El estado de San Luis Potosí ocupa tres escalones altitudinales que en promedio oscilan en el Altiplano 1,800 m, la Zona Media $800 \mathrm{~m}$ y la Planicie Costera del Golfo de México con $200 \mathrm{~m}$. Además, desde el punto de vista climatológico, el estado es atravesado por el Trópico de Cáncer ( $\left.23^{\circ} 27^{\prime} \mathrm{LN}\right)$ y, por lo mismo, presenta características propias de una región intertropical. La región del Altiplano y parte de la región
Media presentan climas semiáridos con lluvias que oscila de los 400 a los $600 \mathrm{~mm}$ de precipitación anual; el sur y sureste del estado presentan climas templados y semicálidos, mientras que la región de la Huasteca presenta un clima tropical subhúmedo con precipitaciones que oscilan de los 800 a los 3,000 mm de lluvia al año.

Gracias a su situación geográfica el estado potosino cuenta con una enorme biodiversidad, en términos biogeográficos, comparte la diversidad florística y faunística del extremo sur del Desierto Chihuahuense con la biodi- 
versidad del extremo norte de las selvas medianas subperennifolias en su porción Huasteca. Su posición en las regiones neotropical (o meridional), boreal (u holártica) y áridas explican sus cortejos florísticos, compuestos por un gran número de especies endémicas y migrantes que han colonizado el territorio potosino en diferentes épocas pretéritas. Esta combinación, que resulta de la localización relativa del estado, explica en parte la riqueza de la biodiversidad del territorio potosino.

Por otra parte, desde la época prehispánica, lo que hoy es San Luis Potosí fue ocupado por grupos indígenas de filiación mesoamericana como los nahuas y tének y grupos de filiación aridoamericana llamados "chichimecas", grupos nómadas que ocuparon una enorme extensión del norte de México e incluía una gran parte del norte del territorio potosino. Por esta razón el estado de San Luis Potosí no sólo posee una naturaleza biodiversa, también presenta una ocupación humana de profundas raíces prehispánicas que ha transformado a la naturaleza primigenia en un mosaico de ambientes de diverso colorido.

Ubicado en la porción centro-norte del país, San Luis Potosí contiene la mayor diversidad de asociaciones vegetales que representan una gran variedad de paisajes naturales. Algunos de ellos han sido aprovechados desde antaño, especialmente los que se ubican en las regiones Media y Huasteca, siendo esta última sin duda la zona de San Luis Potosí la que cuenta con el más alto potencial para un desarrollo sostenible ecoturístico. En esta región encontramos una rica tradición indígena donde diversas lenguas indígenas todavía se hablan normalmente. Esta riqueza cultural abarca hermosas vestimentas, buena comida y tradiciones que se remontan muchos años. Además en los municipios de la región Huasteca se localizan lagunas cascadas y reservas naturales; en complemento, la herencia histórica de la antigua organización territorial se identifica en zonas arqueológicas como Tamtok, perteneciente a la cultura teenek, además como complemento a lo anterior, se localizan también restos de numerosas haciendas que datan de la época colonial presentes en varios municipios del estado.

En la región del Altiplano se localiza el municipio de Real de Catorce que, desde hace más de 20 años se ha consolidado como un ícono turístico nacional y se proyecta como el sitio más reconocido del Altiplano Potosino en el ámbito nacional e internacional. Abarca una porción de la Sierra de Catorce claramente definida y "el Bajío" de Catorce. Los antecedentes históricos lo sitúan como un centro minero que alcanzó su máximo esplendor durante el periodo colonial y, posteriormente, adquirió la apariencia de un lugar deshabitado cuando decayó la producción de plata; aún así El Real nunca ha estado completamente abandonado y la fama de pueblo fantasma se debe a que así lo parecía hasta hace poco, pues la quietud y el silencio eran verdaderamente notables. Actualmente, aunque en él viven tan sólo poco más de 1000 residentes, el cambio en su fisonomía se debe al desarrollo de una intensa actividad comercial y turística que ha revitalizado al municipio de Real de Catorce.

La categoría preferencial que goza Real de Catorce se manifestó en el anuncio que la Secretaría de Turismo hizo a finales de 2004, cuando dio a conocer que para 2005 se invertirían 4.3 millones de pesos en la localidad, con el objeto de "preservar la belleza y el misticismo de un pueblo espectacular". Entre otros rubros, estos recursos se emplearían en la rehabilitación de la red de agua potable, apuntalar el túnel de Ogarrio, el cual permite el acceso

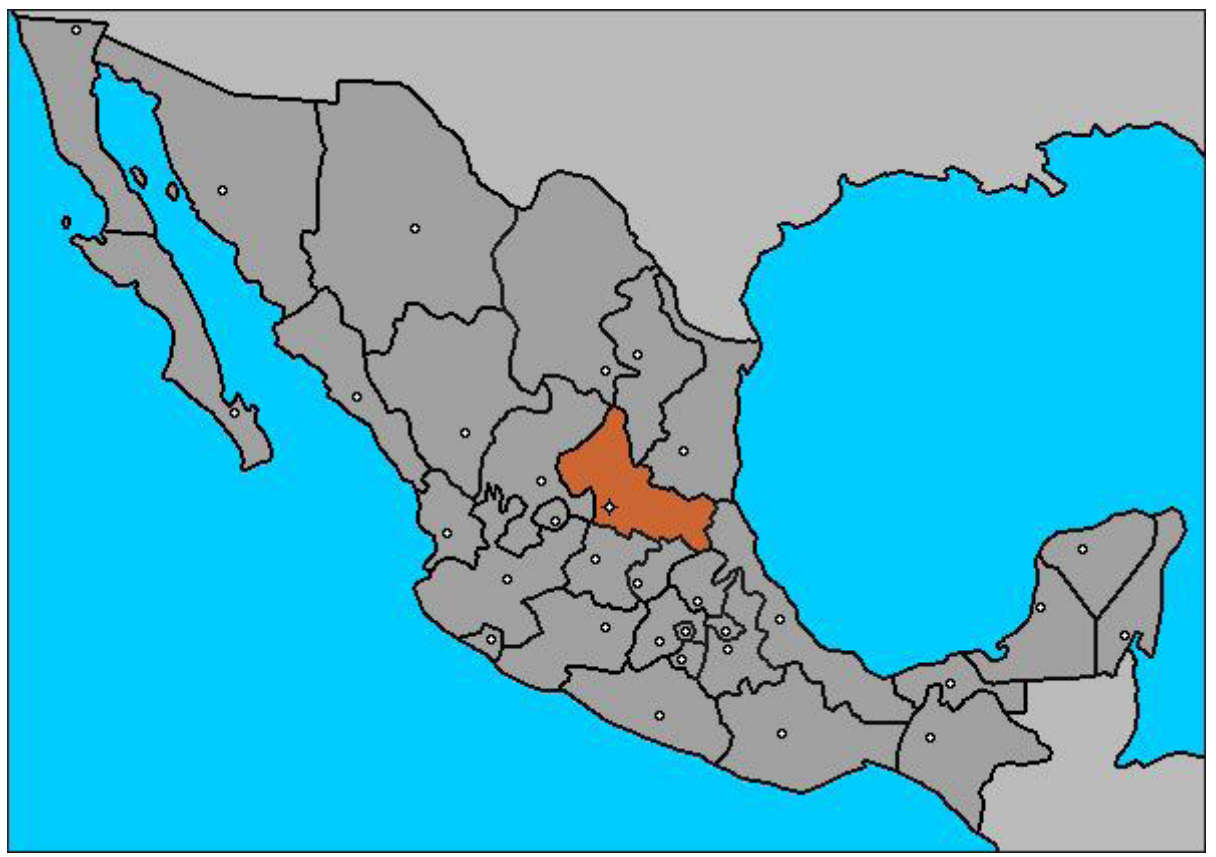

Imagen 1. Ubicación geográfica de San Luis de Potosí . 
al poblado a través de un cerro y el desarrollo de obras dirigidas a conservar el patrimonio arquitectónico y regenerar la imagen urbana con el fin de hacer más atractivo este lugar para los turistas. Por añadidura, se esperaba que más de 30,000 personas residentes en localidades vecinas serían beneficiadas de manera indirecta (Gobierno del estado de San Luis Potosí, 2004).

Poco después, en el tianguis turístico celebrado en Acapulco en abril de 2005, el director general de Fondo Nacional de Fomento al Turismo (FONATUR) John McCarthy, estableció el compromiso de realizar un proyecto de desarrollo turístico integral en la región del Altiplano Potosino mediante el incremento de recursos federales dirigidos a evaluar la infraestructura de transporte y comunicaciones, la factibilidad del suelo y la disponibilidad de agua, entre otros aspectos. De manera paralela, la Secretaría de Turismo del estado ha contemplado la ejecución de dos proyectos turísticos que involucran algunos de los atractivos turísticos reconocidos en la zona.

El primero de ellos es la Ruta de Real de Catorce, cuyas estrategias incorporan el acondicionamiento de pueblos fantasmas para ser visitados, así como la observación del paisaje de matorral xerófilo y la fauna local. Por su parte, la Ruta del Mezcal adquiere importancia en la medida que abarca en el proyecto las mezcaleras ubicadas en la región, correspondientes a los municipios de Charcas, Villa de Guadalupe y Villa de Arista, síntesis que amalgama el legado histórico de las grandes haciendas con la producción artesanal de mezcal. No obstante las condiciones descritas, en 2004, poco más de un millón de turistas nacionales visitaron San Luis Potosí, y menos del $0.5 \%$ de los visitantes internacionales que arribaron a México concurrieron a la entidad, comportamiento sintomático de la escasa o nula correspondencia que establece entre la potencialidad turística y la afluencia efectiva de visitantes $\mathrm{y}$, por consiguiente, la menor preferencia de los turistas para visitar a San Luis Potosí respecto a otros estados del país, los cuales registran los mayores índices de turistas. Por lo tanto, la combinación eficiente en el aprovechamiento de los atractivos se puede convertirse en el mejor detonador para poner en marcha un conjunto de estrategias vinculadas al ecoturismo, actividad que podría, por otra parte, consolidarse como un complemento significativo de los objetivos estratégicos y programas desarrollados por instancias gubernamentales en distintos niveles. Así, para el gobierno del estado, el fortalecimiento del sector turístico, es concebido como un impulsor del desarrollo económico y el generador activo de empleos locales bien remunerados, que permitan la equitativa distribución del ingreso y que se aprovechen los recursos naturales de manera sustentable.

En este sentido, algunos resultados que arrojan recientes investigaciones realizadas en diversas localidades de la entidad, dan cuenta de la variedad y riqueza de los recursos naturales y culturales presentes en las regiones, mismos que son fuente de proyectos turísticos y oportunidades para que las comunidades estén haciendo un uso responsable de sus recursos y de esta manera contribuir al desarrollo de oportunidades para poder impulsar el desarrollo humano a nivel individual y a nivel de las comunidades. Sin embargo, los recursos y el potencial que puedan tener las regiones y algunas de sus localidades requieren desarrollar un intenso proceso de seguimiento y monitoreo de todos los aspectos que se encuentran involucrados en la puesta en marcha de estos proyectos. Es decir, se hace necesario relacionar los recursos naturalesambientales con los intereses económicos, los interés políticos de los agentes locales y de los agentes extralocales (Rangel Valadés, Norma Lucero 2011; Pineda Manzano, Ulises 2010).

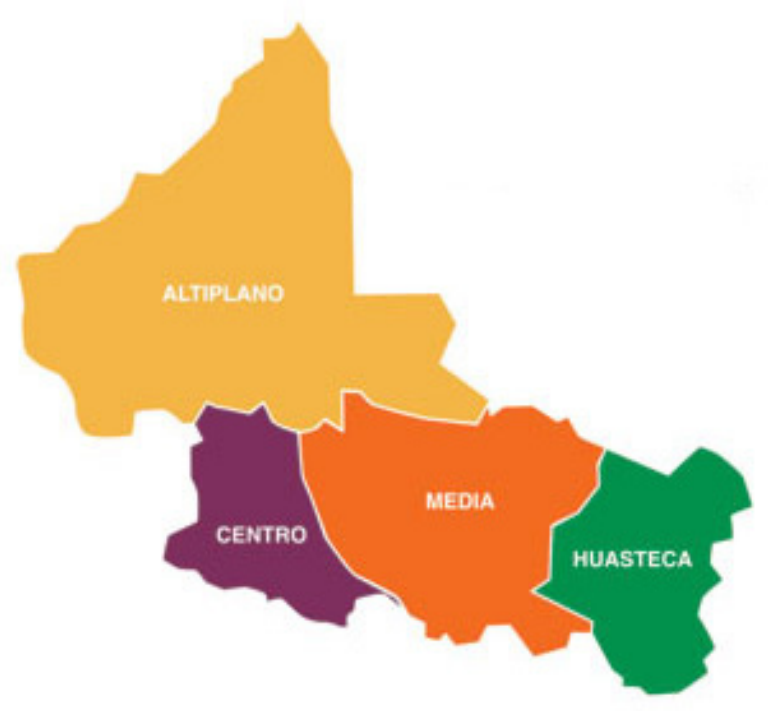

Imagen 2. Localización de las 4 regiones que conforman el territorio potosino.

\section{Componentes del subdesarrollo socioeconómico en San Luis Potosí}

Es un hecho que la pobreza es una realidad evidente en la mayoría del territorio rural y urbano en San Luis Potosí, resultado de las inequidades que distinguen a las actividades productivas y sociales. La situación de pobreza no es una situación que le resulte ajena y desconocida a la población potosina. La situación anterior se expresa entre las personas por la diferencia en oportunidades de trabajo e ingreso; en las microrregiones por las ventajas de unas sobre otras en un contexto de competitividad económica; y entre sectores productivos por los desequilibrios que presenta la economía rural respecto de las actividades industriales y de servicios en el medio urbano.

Al final del sexenio 2003-2009, de un total de 2,410,414 habitantes en el Estado, 3.5\% vivían en 4 municipios considerados de muy alta marginación y el $32.7 \%$ en 37 municipios con marginación alta, lo que significa que más de 800 mil personas vivían en ese entonces en condiciones de rezago social y económico crítico. Se tienen además 
10 municipios con marginación moderada donde vive el $12.3 \%$ de la población; en tanto que poco más de la mitad (51.5\%) reside en 7 municipios de marginación baja y muy baja. Además la marginación, refleja el grado de dificultades que tienen las personas para incorporarse al progreso de su localidad y municipio, por su rezago educativo, por la carencia de servicios básicos en sus viviendas, por sus bajos ingresos y su residencia en pequeñas localidades. Por otra parte, datos recientes del Consejo Nacional de Evaluación de la Política de Desarrollo social (CONEVAL, 2009), señalan que el $15.2 \%$ de la población se encuentra en pobreza extrema; el $35.9 \%$ en pobreza moderada y que en total más de la mitad de la población, el 51.1\% padece pobreza, lo que significa un millón 260 mil personas. En materia de desarrollo humano, de acuerdo con información del Programa de Naciones Unidas para el Desarrollo (PNUD, 2006), San Luis Potosí ocupa en lugar 19 en el contexto nacional por su índice de desarrollo humano, resultado de sus limitadas posibilidades para que la población alcance un nivel de vida saludable; conocimientos individuales y socialmente valiosos; y la oportunidad de obtener los recursos necesarios para disfrutar de un nivel de vida decoroso (SEDESORE/SLP, 2010).

La promoción y planeación del turismo en San Luis Potosí desde la perspectiva gubernamental

Los planteamientos en torno al desarrollo del turismo en la entidad quedaron plasmados en el Plan Estatal de Desarrollo 2003-2009. En esa ocasión, el enfoque gubernamental se concentró en los siguientes objetivos:

- Impulsar y consolidar el potencial económico del turismo: la industria turística debe ser impulsada con acciones que aseguren la sustentabilidad de los sitios, evitando la depredación.

- Mejorar la imagen y el paisaje urbano de cada municipio: todos inclusive aquellos municipios que no son considerados sitios turísticos, pueden maximizar su potencial con proyectos dirigidos a mejorar la imagen urbana y la infraestructura.

De lo anterior, se propusieron las siguientes estrategias para alcanzar los propósitos concebidos en el Plan Estatal de Desarrollo 2003-2009:

- Elaborar un programa estratégico para el turismo con una visión a largo plazo.

- Elaborar un inventario de los sitios con potencial turístico.

- Fortalecer y promover el turismo, la cultura y los sitios con atractivos naturales bajo el principio de desarrollo sustentable; considerando y respetando los usos y costumbres de los habitantes de las comunidades y regiones del estado.

- Mejorar la imagen urbana de los municipios así como resguardar y conservar su patrimonio histórico y arquitectónico.

Otro documento fundamental para la planeación del turismo en la entidad fue el Programa Sectorial de Turismo. De este documento a continuación se enuncian los objetivos y las estrategias del programa.

Objetivo 1: Impulsar el desarrollo de los servicios básicos en lugares con potencial turístico.

\section{Estrategias:}

- Desarrollar y ejecutar, proyectos turísticos socialmente rentables.

- Promover y gestionar el desarrollo de servicios de apoyo o complementarios a la oferta turística.

- Generar un banco de datos estatales de sitios con potencial turístico y determinar su vocación turística.

Objetivo 2: promover las inversiones y e financiamiento al sector turístico para que lleve a un crecimiento balanceado y al fortalecimiento de la oferta turística.

\section{Estrategias:}

- Promover las inversiones privadas en sitios en los cuales puedan ser exitosas.

- Inducir una política estatal integral para el financiamiento del sector turístico.

- Impulsar la generación de MPYMES turísticas y facilitar e acceso a financiamiento.

Objetivo 3: personalizar y hacer eficiente la administración pública del sector turismo.

\section{Estrategias:}

- Generar información, estudios y análisis útiles para una optima administración pública y el mejor entendimiento del sector.

- Garantizar que la operación de la administración pública este dirigida a alcanzar las metas y que estas se logren con la mejor calidad y un mínimo costo posible.

Los anteriores se establecieron como los objetivos de la administración estatal durante el sexenio 2003-2009, periodo, que como ya se comentó anteriormente, se desarrollaron importantes proyectos para la promoción del turismo en las diferentes regiones del estado. No obstante la práctica del turismo en la entidad sigue enfrentando importantes retos y obstáculos, mismos que tendrán que atenderse por cada uno de los actores involucrados en esta actividad. En este sentido, a continuación se presentaran algunos de los resultados generados a partir del trabajo de campo que se realizó en diferentes municipios de tres de las cuatro regiones que conforman el estado de San Luis Potosí (Región Altiplano, Media y Huasteca).

\section{Fortalezas y debilidades del turismo y ecoturismo} en San Luis Potosí

El trabajo de campo incluyó 11 municipios de las regiones Altiplano, Media y Huasteca . En los municipios en donde se llevó a cabo la investigación se tuvo la posibilidad de contactar con las autoridades municipales (encargados o responsables municipales de las áreas de turismo y también a los tres representantes regionales del gobierno estatal en materia de turismo). Cabe destacar que la principal intención de esta actividad consistió en recabar información que permitiera al equipo de trabajo conocer de cerca las principales problemáticas referentes a la actividad turística desde la perspectiva de quienes tienen la 


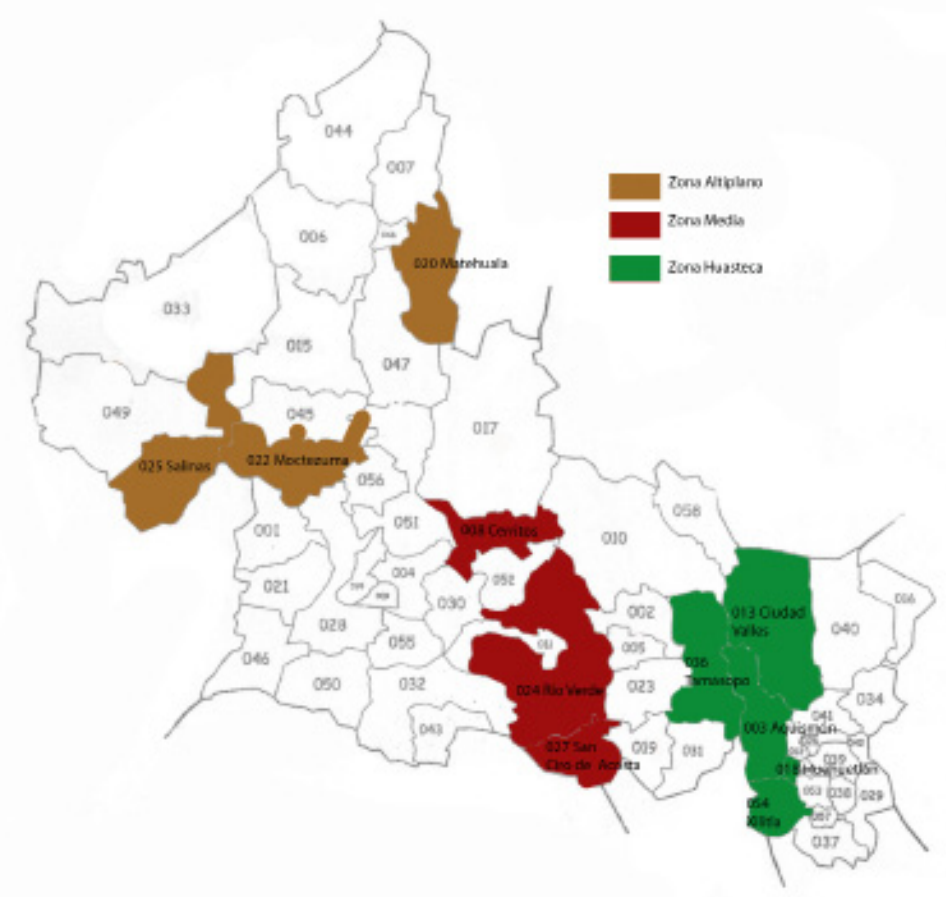

Imagen 3. Ubicación de los 11 municipios en los que se realizaron las entrevistas y el trabajo de campo en el proyecto de investigación.

posibilidad de tomar decisiones. De este modo, se obtuvo una categoría perceptiva adicional que ofrece un contexto mucho más amplio y certero del problema examinado, en este caso, la situación de la actividad turística y ecoturística en el estado de San Luis Potosí.

Del análisis de la información contenida en las entrevistas realizadas a los funcionarios se destacan los argumentos indicados en el cuadro 3 .

\section{Apuntes finales}

Frente a las situaciones de pobreza y marginación que enfrenta la mayoría de la población y las regiones del estado de San Luis Potosí, la promoción del turismo y el ecoturismo se presenta como una alternativa real para abatir estas condiciones de rezago entre la población afectada. Lo anterior se vuelve relevante debido a que las regiones estudiadas cuentan con un potencial humano, cultural y natural para la instrumentación de proyectos enfocados a alcanzar esta meta. Sin embargo, lo que aquí queremos destacar es que el ejercicio de la planeación de los proyectos y estrategias para implementar nuevas opciones de desarrollo requiere considerar varias posibilidades que al momento de considerar la planeación son dejadas de lado. En este sentido, si se pretende generar estrategias que estén encaminadas en volver competitivo algunas regiones del estado en mate- ria de proyectos relacionados con el turismo y el ecoturismo, sin duda que se deben de considerar los aspectos que se han recogido en voz de los actores sociales y de las autoridades a nivel local y a nivel regional. Ya en otros trabajos (Valente, et. al.; 2010; Reyes Hernández, et. al,; 2009), señalamos algunas consideraciones fundamentales para el desarrollo del turismo y el ecoturismo en diversas regiones del propio estado. Sin embargo, derivado de la investigación que abarco 3 regiones más del estado, estamos en condiciones de emitir las siguientes recomendaciones al respecto:

- La necesidad de promover estrategias encaminadas a promover el trabajo coordinado entre las diversas autoridades relacionadas con el turismo y el ecoturismo (federal, estatal y municipal), de tal manera que se establezcan programas que verdaderamente fomenten una participación y un dialogo transversal entre los diferentes ordenes de gobierno.

- Estimular una constante y permanente capacitación de todos los sectores y actores involucrados en la instrumentación y gestión de proyectos relacionados al turismo. En este sentido, la capacitación se vuelve un aspecto fundamental para miembros de las comunidades que se han visto involucradas en este proceso, ya que son las propias comunidades las que deben promover y echar a andar sus propios proyectos y establecer los mecanismos de seguimiento. Es decir, son las comunidades las encargadas de establecer sus propios 


$\begin{aligned} & \text { Fortalezas del turismo y ecoturismo en el estado } \\ & \text { de San Luis Potosí }\end{aligned}$
En los recorridos realizados en las regiones de estu-
dio, pudimos constatar la gran diversidad de pai-
sajes naturales y actividades culturales que pueden
ser promovidas y aprovechadas como generadores
de oportunidades para el turismo y el ecoturismo.

En algunas localidades los miembros de la comunidad son los responsables de elaborar sus propias propuestas de manejo y de conservación del recurso natural, además son las propias comunidades quienes proponen sus normas en función de los intereses que atañan a la población; también se ha buscado que dicha normativa sea ajena a la burocracia que tradicionalmente es impuesta desde la comodidad y la arrogancia de un escritorio que, con frecuencia, planea los proyectos de desarrollo turístico sin considerar o tomar en consideración un conjunto de variables de carácter cultural.

En varias localidades pertenecientes a los municipios estudiados se ha logrado la consolidación de algunos grupos y proyectos exitosos que permitirían la replicación de su experiencia en otras comunidades de la entidad

Algunos proyectos han sido sensibles al ámbito sociocultural de las comunidades, es decir se han puesto en marcha acciones y proyectos que ser acordes con lo que la tradición marca, y que enfaticen especialmente en respetar su propia organización y sus costumbres. En este sentido, ha sido muy importante contar con un respaldo comunitario y eso solamente se logra en el momento en que las comunidades son, involucradas y tomadas en cuenta.

En algunos parajes de los municipios de la región Huasteca se ha promovido la formación de los llamados consejos de turismo, en ellos se trata de dar solución a los conflictos que se generan al interior de las comunidades por la manera en la que se pretende realizar el aprovechamiento de los recursos. Resultado de las gestiones que realizan estos llamados consejos de turismo es que se ha logrado dar solución a muchas de las situaciones que enfrenta a las comunidades. Un avance es que en algunos casos se ha logrado la creación de reglamentos internos para la operación de algunos parajes, y estos reglamentos han sido fundamentales para hacer un uso más responsable de sus recursos

\section{Debilidades del turismo y ecoturismo en el estado} de San Luis Potosí

En los recorridos y en las pláticas con las autoridades y con las personas de las comunidades, fue posible darnos cuenta de la existencia de diversos conflictos entre las propias comunidades por la falta de definición sobre límites territoriales, lo cual ha llevado en ocasiones a que se generen una serie de disputas entre los pobladores de diferentes comunidades, creyendo que solo algunos tienen el derecho de verse beneficiados por la presencia o llegada de los turistas.

En algunas localidades que cuentan con recursos naturales y culturales que propician la práctica del turismo, hay una ausencia de programas o proyectos que coordinen los intereses de las comunidades. Por ejemplo algunos miembros de la localidades han tomado el derecho de cobrar por la entrada a ciertos lugares, cuando esa actividad en muchos casos no está reglamentada por la comunidad o parte de ello, lo cual termina generando situaciones que enfrenta a la población por la posesión y el derecho a usufructuar los recursos existentes en el territorio.

En la mayor parte de las entrevistas fue evidente el hecho de que en los municipios y en las organizaciones comunitarias no se cuenta con la información pertinente, además no se promueve o se llevan a cabo estrategias de capacitación para ofrecer servicios, y tampoco se da supervisión o asesoramiento necesario. Como resultado de las deficiencias o carencias anteriores, los daños ecológicos en algunos parajes son tan evidentes, lo cual se desarrolla de forma paralela al enfrentamiento entre las personas, incluso en una misma comunidad.

Se resaltó en los recorridos y en las entrevistas el hecho de que en la mayoría de los sitios con potencial turístico no cuentan con Planes de Manejo Integrales, en los que se definan estrategias o políticas claras de implemento de la actividad turística o ecoturística, en donde se consideren los problemas sociales, ambientales, y la organización territorial de la actividad bajo un esquema de ordenamiento ecológico integral.

Es evidente la falta capacitación a la gente de las comunidades y de los prestadores de servicios de las regiones del estado para que puedan brindar un servicio de calidad al visitante que llega a los municipios y a las localidades en las temporadas de vacaciones. Mucha gente de las comunidades que poseen algún recurso natural y cultural que pueda ser utilizado en beneficio de las mismas ve en el turismo y el ecoturismo una posibilidad de poder generar recursos para mejorar la calidad de vida; sin embargo estas comunidades y muchos de sus miembros carecen de capacitación en el manejo del recurso ambiental o de los recursos financieros, capacitación que les sería útil para lograr verdaderos cambios en su calidad de vida. 
Fortalezas del turismo y ecoturismo en el estado de San Luis Potosí

En algunas localidades existen incipientes vínculos entre los sectores educativo-académico y empresarial. Estos avances, no obstante que son incipientes, pueden ser significativos ya que el primero tiene la capacidad de generar resultados investigativos viables para su aplicación en materia turística con base en propuestas metodológicas sólidas y científicas, y con el segundo se pueden crear sinergias que favorezca la inversión regulada en el fomento a la actividad turística local, regional y estatal. En particular sobre este último aspecto, es un hecho que los inversionistas privados estén ausentes en los municipios en la región, la inversión en infraestructura hotelera es prácticamente nula y solo las autoridades del estado se hacen presentes con presupuestos de gasto corriente e inversión marginales. Incluso, cuando ha ocurrido, las inversiones realizadas en el estado han sido generadoras de conflictos en virtud de que privilegia determinados sectores de población, o bien, se dirige a sitios específicos.

En algunos proyectos turísticos, las mujeres de las comunidades se han constituido como el núcleo central en la vida de las comunidades y han sido un elemento detonante en el éxito que han tenido algunas propuestas llevadas a la práctica.

La renovada presencia de instituciones de educación media y superior en la región Centro y Huasteca ha podido hacer algunos avances en la capacitación y profesionalización del personal que participa de muy diversas formas en las actividades relacionadas el desarrollo del turismo, en este sentido esto representa un importante avance, ya que se abre una oportunidad para que los cargos públicos o privados que estén vinculados o relacionados con la práctica del turismo sean ocupados por personas que cumplan con un perfil profesional que responda a las necesidades y a los retos de la región y de las comunidades.
Debilidades del turismo y ecoturismo en el estado de San Luis Potosí

En la mayoría de las comunidades estudiadas, cada actor o sector involucrado termina realizando una parte significativa de sus actividades de forma individual, fragmentada e incluso opuesta a la forma de aprovechamiento de largo plazo, o bien sustentable. Es de destacar que a partir de este ejercicio de acercamiento y de dialogo con las autoridades municipales y regionales, se pudo dar cuenta de que tampoco se cuenta con estrategias a mediano y largo plazo emanadas de las autoridades y extensivas a la población, y que en ellas se propongan una serie de medidas de conservación de áreas naturales, tanto en aquellas que ya son utilizadas como de otras que podrían incorporarse, en el corto o en el mediano plazo, como parte de la oferta turística local, regional y estatal.

Los responsables a nivel municipal, destacaron la falta de apoyo del gobierno estatal. Por ejemplo destacaron que no se les apoya con infraestructura básica (computadoras, material de papelería, trípticos) para que los funcionarios municipales realicen sus actividades de gestión o de planeación. El gobierno estatal exige que se fomente y se desarrolle el turismo, sin embargo la realidad cotidiana es otra. En este sentido se destaca la necesidad de fomentar estrategias que privilegien el trabajo de forma coordinada entre las propias autoridades municipales, porque muchas veces se anteponen los intereses de alguna dependencia que dañan o perjudican lo realizado por otras, quienes pretenden alcanzar o consolidar una estrategia de verdadera planeación en la ejecución de una actividad turística con un sentido ambiental y socialmente responsable. En general hace falta mayor coordinación entre las instancias involucradas. A manera de ejemplo, se comenta.

No hay coordinación entre los distintos niveles de decisión político-administrativa del estado y los municipios, pues normalmente estos últimos manifiestan el escaso o nulo apoyo obtenido por parte de las autoridades estatales y, especialmente, de las instancias federales que incluso ignoran por completo las condiciones y problemas asociados con el desarrollo turístico a esta escala de manifestación. 
Cuadro 3 (Continuación). Fortalezas y debilidades del turismo y ecoturismo en San Luis Potosí (continuación)

\begin{tabular}{|l|l|}
$\begin{array}{l}\text { Fortalezas del turismo y ecoturismo en el estado } \\
\text { de San Luis Potosí }\end{array}$ & $\begin{array}{l}\text { Debilidades del turismo y ecoturismo en el estado } \\
\text { de San Luis Potosí }\end{array}$ \\
\hline & $\begin{array}{l}\text { La existencia de desigualdades socioeconómicas al in- } \\
\text { terior de las comunidades hace que esto genere des- } \\
\text { igualdades en el manejo de oportunidades y en el ma- } \\
\text { nejo de los recursos que se logran captar para algunos } \\
\text { proyectos en particular. Normalmente los que tienen } \\
\text { más terrenos siempre tendrán mas oportunidades y } \\
\text { los que cuenten con otros recursos siempre buscan abu- } \\
\text { sar de los que no tienen o tienen menos. Por esta ra- } \\
\text { zón, hace falta crearles a las comunidades el habito de } \\
\text { que es posible que puedan vivir de la actividad turísti- } \\
\text { ca, hace falta que se identifiquen más con sus propios } \\
\text { valores, con su entorno y que aprendan a aprovechar } \\
\text { los recursos naturales, sociales, históricos y cultura- } \\
\text { les que se encuentran presentes en sus comunidades. }\end{array}$ \\
& $\begin{array}{l}\text { Las carreteras se encuentran en condiciones inade- } \\
\text { cuadas, y la señalética, además de insuficiente, es con- } \\
\text { fusa en ocasiones. Sin embargo, el gobierno limita con } \\
\text { frecuencia los apoyos para la dotación de estos servi- } \\
\text { cios, en virtud de los problemas que significa favorecer } \\
\text { determinadas comunidades por sobre aquellas que no } \\
\text { cuentan con atractivos turísticos que incentiven la in- } \\
\text { versión en carreteras y señalética con fines turísticos. }\end{array}$ \\
\hline
\end{tabular}

Fuente: Elaboración de los autores a partir del trabajo de campo realizado en el marco del proyecto: "Potencial natural y socioeconómico para

el desarrollo del ecoturismo en el estado de San Luis Potosí.

proyectos y sus propios reglamentos, sin embargo ese es un proceso largo y desgastante.

- Considerar las variables culturales, étnicas, económicas y hasta de género que se presentan en la entidad y que son fundamentales para alcanzar las metas que puedan convertirse en los detonadores del desarrollo.

- El turismo y el ecoturismo deben ser una estrategia ambiental y socialmente responsable, enfocada a mejorar las condiciones de vida, pero respetando el recurso natural y cultural y buscando con ello superar las brechas de la desigualdad presentes en las 4 regiones de la entidad potosina..

- Fomentar una mayor participación y vinculación entre el sector turístico, las comunidades y el sector académico. De tal forma que los resultados de las investigaciones que se emprendan por los investigadores de diversas instituciones de educación superior puedan impactar en la generación de mejores condiciones de vida a partir del aprovechamiento de los recursos naturales y culturales de las comunidades.

Como se señaló con anterioridad, las autoridades estatales han visualizado al turismo y al ecoturismo como un motor de oportunidades, pero para que esto se haga realidad se tienen que considerar algunos de los puntos que aquí se han mencionado. Además hay que considerar al turismo y al ecoturismo como una estrategia dentro de muchas más que se deben de estar considerando al interior de las regiones. Es decir, el turismo y el ecoturismo por si mismos no deben de ser la única alternativa para alcanzar el desarrollo regional que se espera.

\section{Bibliografía}

Genet, Guzmán Chávez, Mauricio

2008 “Qué se busca con el ecoturismo?” Gaceta Cienci@ San Lis Potosí. Mx. Órgano de difusión científica de la Universidad Autónoma de San Luis Potosí, El Colegio de San Luis, el Consejo Potosino de Ciencia y Tecnología y el Instituto Potosino de la investigación Científica y Tecnológica. Año 3, Número 34.

Gobierno del estado de San Luis Potosí

2004 Programa Sectorial de Turismo del Gobierno del estado de San Luis Potosí. San Luis Potosí, México

López Santillán, Ángeles y Gustavo Marín Guardado

2010 "Turismo, capitalismo y producción de lo exótico: una perspectiva crítica para el estudio de la mercantilización del espacio y la cultura”, Revista Relaciones 123, Vol. XXXI. Pp 219-258

Marín Guardado, Gustavo

2006 "Desarrollo turístico, territorialidad y configuración socioeconómica en la riviera maya". Revista Ichan Tecolotl. CIESAS. Febrero 2006, Año 16, Núm. 186. 
Programa de las Naciones Unidas para el Desarrollo (PNUD)

2006 Informe sobre Desarrollo Humano. San Luis Potosí 2005. Programa de las Naciones Unidas para el Desarrollo, México, D.F.,

Pierce, D.

1995 Tourism today. A Geographical Analysis, Second edition. Longman. Harlow, England

Pineda, Manzano Ulises.

2010. Ecoturismo en la zona media del estado de San Luis Potosí: una propuesta de diversificación económica. Tesis para optar por el título de licenciado en Geografía. San Luis Potosí.

Rangel Valadés Norma Lucero.

2011. Propuesta de turismo sustentable en el municipio de Guadalcázar, San Luis Potosí. Tesis para optar por el título de licenciado en Geografía. San Luis Potosí

Reyes, Hernández Humberto, et. al.

2009. "Impacto ambiental de las actividades ecoturísticas en el estado de San Luis Potosí, México". Memorias del VIII Seminario de Investigación Urbano-regional. Universidad Nacional de Colombia, sede Bogotá.

Rivera, González, José Guadalupe

2005 "Acapulco y Ciudad Renacimiento: efectos del turismo en el pacifico mexicano". Ponencia presentada en el marco del Seminario de Investigación: Turismo y Ecoturismo, evento realizado en las instalaciones de la $\mathrm{CCSyH}$

Sánchez, A.

2001 "El territorio y nuevas formas del turismo en México: el caso de Mapimí, Durango". Memorias del XVI Congreso Nacional de Geografía, Mérida 2001. INEGI, Aguascalientes, México. pp. 628-640

Secretaría de Desarrollo Social del Gobierno del Estado de San Luis Potosí

2010 Diagnostico Sectorial de Desarrollo Social. San Luis Potosí, México.

Vázquez, V. y Benito, P.

2009 "Turismo y territorio en México: alternativas en el Altiplano Potosino". Biblio. Revista Bibliográfica de Geografía y Ciencias Sociales, Universidad de Barcelona, Vol. XIV, no 813, 25 de febrero de 2009.

Vázquez, Solís Valente

2008 "Ecoturismo: entre la práctica económica y la cultura responsable" Gaceta Cienci@ San Lis Potosí. Mx. Órgano de difusión científica de la Universidad Autónoma de San Luis Potosí, El Colegio de San Luis, el Consejo Potosino de Ciencia y Tecnología y el Instituto Potosino de la investigación Científica y Tecnológica. Año 3, Número 34.

Vázquez, Valente, et. al

2010 "Evaluación de los atractivos naturales para el desarrollo del ecoturismo en la región Huasteca de San Luis Potosí, México" Cuadernos de turismo. Universidad de Murcia. No. 25
Vera, J.F y A. López Marchena

1997 Análisis territorial del Turismo. Ariel, Barcelona, España.

Fuentes consultadas en Internet:

http://www.explorandomexico.com.mx/about-mexi$\mathrm{co} / 5 / 137$

\section{Nota}

${ }^{1}$ Este artículo se desprende de un proyecto de investigación más amplio denominado: Potencial natural y socioeconómico para el desarrollo del ecoturismo en el estado de San Luis Potosí. Este proyecto contó con el financiamiento del Gobierno del Estado de San Luis Potosi y del Consejo Nacional de Ciencia y Tecnología (CONACYT), a través de los denominados Fondos Mixtos - San Luis Potosí (FOMIXSLP). En dicho proyecto se conformo un equipo multidisciplinario conformado por 1 antropólogo, 5 geógrafos y 1 arqueólogo. Además se incorporaron a un núcleo de estudiantes de las mismas licenciaturas arriba señaladas para apoyar en las actividades de recolección y organización de la información generada en gabinete y en campo.

Recibido:

$23 / 09 / 2010$

Reenviado:

$03 / 04 / 2011$

Aceptado:

$21 / 10 / 2011$

Sometido a evaluación por pares anónimos 